\title{
Fechamento Percutâneo da Comunicação Interventricular Muscular Congênita
}

\author{
Carlos A. C. Pedra ${ }^{1,2,3}$, Simone R. F. Pedra ${ }^{1,2}$, Cristiane Pessotti ${ }^{1}$, Maria Virginia T. Santana ${ }^{2}$, \\ leda Jatene', Mônica Shimoda ${ }^{1}$, César A. Esteves², Sérgio L. N. Braga ${ }^{2}$, \\ Maria Fernanda Jardim ${ }^{3}$, Justo Santiago ${ }^{4}$, Valmir F. Fontes ${ }^{1,2}$
}

\section{RESUMO}

Introdução: O tratamento percutâneo da comunicação interventricular (CIV) congênita vem sendo realizado com bons resultados nos últimos 10 anos. Relatamos nossa experiência com a oclusão percutânea da CIV muscular congênita com próteses Amplatzer, avaliando exeqüibilidade, segurança e eficácia do método. Método: No período de setembro de 2002 a dezembro de 2007, foram realizados 9 procedimentos em múltiplos centros em 8 pacientes não-consecutivos (mediana de idade de 6 anos e de peso de $26 \mathrm{~kg}$ ), sob anestesia geral e monitoração pela ecocardiografia transesofágica. Todas as CIVs eram únicas (7 na região trabecular média e 1 na porção anterior) e tinham diâmetro médio de $6,0 \pm 2,1 \mathrm{~mm}$. Houve embolização imediata de um dispositivo com resgate percutâneo. O procedimento foi repetido com sucesso após um ano. Todos os casos restantes foram realizados com sucesso, sem complicações maiores. O índice de oclusão foi de $100 \%$ no seguimento. Conclusão: Nessa pequena série de pacientes, a oclusão percutânea da CIV muscular congênita única com próteses Amplatzer foi um procedimento de fácil execução, seguro e altamente eficaz.

DESCRITORES: Defeitos do septo interventricular/terapia. Próteses e implantes. Oclusão com balão. Cateterismo.

A comunicação interventricular (CIV), entre as cardiopatias congênitas, é a mais freqüente, correspondendo a cerca de $25 \%$ de todas elas ${ }^{1}$. O tipo mais comum é a perimembranosa, seguido da muscular, da de via de saída e da de via de entrada ${ }^{1,2}$.

\footnotetext{
1 Hospital do Coração - Associação do Sanatório Sírio - São Paulo, SP.

2 Instituto Dante Pazzanese de Cardiologia - São Paulo, SP.

${ }^{3}$ Hospital Samaritano - São Paulo, SP.

4 Hospital Universitário de Los Andes - Mérida, Venezuela. Correspondência: Carlos A. C. Pedra. Chefe da Seção Médica de Intervenções em Cardiopatias Congênitas. Instituto Dante Pazzanese de Cardiologia. Av. Dr. Dante Pazzanese, 500 - 14 andar - São Paulo, SP - CEP 04012-180 - Tel.: (11) 5085-6114 - Fax: (11) 5085-6196 E-mail: cacpedra@uol.com.br

Recebido em: 10/3/2008 • Aceito em: 26/5/2008
}

\section{SUMMARY}

Percutaneous Closure of Congenital Muscular Ventricular Septal Defects

Background: Percutaneous treatment of muscular ventricular septal defects (VSD) has been performed with good results in the last 10 years. We report our experience with the percutaneous closure of congenital muscular VSDs with Amplatzer devices assessing the feasibility, safety and efficacy of the procedure. Methods: From 9/2002 to 12/ 2007, 9 procedures were performed in multiple centers in 8 non-consecutive patients (median age: 6 years; median weight: $26 \mathrm{~kg}$ ) under general anesthesia and transesophageal echocardiographic monitoring. All VSDs were single ( 7 in the trabecular region and 1 anterior) and had a mean diameter of $6.0 \pm 2.1 \mathrm{~mm}$. There was one device embolization with immediate percutaneous device retrieval. This procedure was successfully repeated after a year. The other cases were completed successfully without major complications. The rate of complete closure was 100\% at follow-up. Conclusion: In this small series of patients, percutaneous closure of congenital single muscular VSD was relatively easy to perform, safe and highly effective.

DESCRIPTORS: Heart septal defects, ventricular/therapy. Prostheses and implants. Balloon occlusion. Catheterization.

A CIV muscular, por definição, está circundada por tecido muscular ao redor de todo o defeito e geralmente fica distante do nó atrioventricular e dos mais importantes feixes de condução ${ }^{1,2}$. Pode estar localizada na região trabecular, com extensão para o ápice, via de entrada ou de saída, na região apical ou na anterior ${ }^{1,2}$. Quando pequena, a história natural mostra boas chances de oclusão espontânea até 4-5 anos de idade $^{1,2}$. Seu tratamento está indicado quando causa insuficiência cardíaca no primeiro ano de vida ou, após essa idade, quando há repercussão hemodinâmica, caracterizada pelo aumento das dimensões do ventrículo esquerdo à ecocardiografia ${ }^{1,2}$. O fechamento oportuno da CIV muscular previne o aparecimento de complicações futuras, como hipertensão arterial pulmonar, insuficiência cardíaca e arritmias ${ }^{1,2}$. 
A correção cirúrgica dessa doença foi introduzida no final da década de 50 e vem sendo empregada com resultados variados, dependendo da localização e do número das CIVs e da idade do paciente ${ }^{2-6}$. O tratamento percutâneo da CIV muscular foi introduzido em 1988 e teve resultados iniciais limitados ${ }^{7-10}$. Nos últimos 10 anos, os resultados têm melhorado significativamente com o uso de próteses intracardíacas de última geração ${ }^{10-22}$.

Em nosso meio, são escassas as publicações relativas à oclusão percutânea das $\mathrm{CIVS}^{23-25}$, especialmente as musculares, o que nos motivou a relatar nossa experiência com esse tipo de procedimento para a CIV muscular única de origem congênita, analisando a viabilidade, a segurança e a eficácia do método.

\section{MÉTODO}

Trata-se de estudo prospectivo, longitudinal e observacional de uma coorte de pacientes submetidos a oclusão percutânea e perventricular da CIV muscular congênita única com próteses intracardíacas, de forma não-consecutiva, em múltiplos centros. Os responsáveis legais pelos pacientes foram adequadamente informados sobre os riscos e benefícios do procedimento e assinaram o termo de consentimento.

\section{Critérios de inclusão}

1. Crianças, adolescentes e adultos, de ambos os sexos, com diagnóstico clínico e ecocardiográfico de CIV muscular única de localização variada (trabecular no meio do septo, apical ou anterior), com repercussão hemodinâmica, caracterizada pelo aumento do ventrículo esquerdo maior que 2 desvios padrão da normalidade para a idade.

\section{Critérios de exclusão}

1. Lactentes com menos de $8 \mathrm{~kg}$;

2. CIVs de outros tipos, incluindo a perimembranosa, a de via de entrada e a de via de saída, também chamada de duplamente relacionada;

3. CIVs musculares com ventrículo esquerdo de dimensões normais;

4. CIVs musculares múltiplas com necessidade de mais de 2 dispositivos;

5. doenças cardíacas associadas com necessidade de intervenção cirúrgica com uso de circulação extracorpórea concomitantemente ao procedimento;

6. doenças infecciosas ativas;

7. alergia comprovada ao níquel;

8. impossibilidade de acesso venoso na veia femoral ou jugular interna;

9. recusa em assinar o termo de consentimento;
10. CIVs musculares de anatomia desfavorável para tratamento percutâneo, muito próximas das valvas atrioventriculares ou semilunares.

O último item dos critérios de exclusão foi definido por meio da ecocardiografia transtorácica, antes do procedimento, e/ou pela ecocardiografia transesofágica, durante o procedimento, e por profissional experiente na seleção dos pacientes para esses tipos de procedimento.

\section{Avaliação clínica pré-procedimento}

Os pacientes foram submetidos a avaliação clínica rotineira, com obtenção de exames laboratoriais, incluindo eletrocardiograma, radiografia de tórax, ecocardiografia transtorácica, hemograma, coagulograma, eletrólitos, glicemia, avaliação da função renal, tipagem sanguínea e urina tipo I.

\section{Dispositivos}

Neste estudo, foram utilizados dispositivos da família Amplatzer (AGA Medical Corporation - Minnesotta, Estados Unidos). O dispositivo para CIV muscular é uma prótese feita de malha de fios ultrafinos de nitinol, composta de 2 discos conectados por cintura central de comprimento de $7 \mathrm{~mm}$ (Figura 1). Os discos possuem diâmetro $8 \mathrm{~mm}$ maior que a cintura central. $\mathrm{Na}$ porção interna da malha de nitinol, encontram-se retalhos de poliéster que aumentam o poder de oclusão do dispositivo. Essa prótese é auto-expansível e autocentrável e é implantada por sistema de baixo perfil que permite resgatá-la e reposicioná-la em qualquer momento até sua liberação final do cabo liberador. Tal cabo conecta-se ao disco do lado direito por um sistema de rosca em um parafuso tipo fêmea. Selecionou-se um dispositivo cuja cintura central, que possui diâmetros de $4 \mathrm{~mm}$ a $16 \mathrm{~mm}$, fosse $1-3 \mathrm{~mm}$ maior que o maior diâmetro do defeito medido no final da diástole. Tal prótese requer o uso de bainhas de baixo perfil para o implante (entre $6 \mathrm{Fr}$ e $9 \mathrm{Fr}$ ). Alternativamente, no caso da não disponibilidade do uso do dispositivo de CIV muscular, utilizaram-se dispositivos Amplatzer para oclusão da comunicação interatrial $(\mathrm{CIA})$ e da persistência do canal arterial (PCA). Nesses casos, o tamanho do corpo central do dispositivo também obedeceu à regra de ser 1-3 mm maior que a CIV. Todos esses dispositivos possuem registro na Agência Nacional de Vigilância Sanitária (ANVISA) e estão liberados para uso clínico no Brasil.

\section{Procedimentos}

Depois de observado o período de jejum de 8 horas, os pacientes realizaram cateterismo cardíaco direito e esquerdo rotineiro no laboratório de hemodinâmica, sob anestesia geral e monitoração da ecocardiografia transesofágica. Após terem sido estabelecidos os acessos venoso e arterial, sulfato de heparina foi administrado na dose de $100 \mathrm{Ul} / \mathrm{kg}$ (máximo de $5.000 \mathrm{UI}$ ) juntamente com cefalotina $(30 \mathrm{mg} / \mathrm{kg}$; máxi- 
mo de $1.000 \mathrm{mg}$ ). Angiografias nas projeções hepatoclavicular e axial alongada foram obtidas para ótimo delineamento do defeito (Figura 2). Para os casos de CIVs musculares trabeculares e apicais, a via de acesso para o implante foi a veia jugular interna direita. Para os casos de CIV muscular anterior, a via de acesso foi a veia femoral. De forma semelhante a protocolos descritos anteriormente $\mathrm{e}^{23-25}$, a CIV foi ultrapassada com cateter Judkins de artéria coronária direita com o auxílio de fio-guia hidrofílico e uma alça arteriovenosa foi estabelecida de acordo com técnicas previamente descritas. Com o suporte da alça arteriovenosa, uma bainha longa tipo Mullins com perfil apropriado para o tamanho da prótese escolhida (6-9 Fr) foi avançada até o ventrículo esquerdo. Após estabilização da bainha longa no corpo do ventrículo esquerdo ou mesmo na aorta ascendente, o guia e o dilatador foram retirados e um dispositivo de tamanho apropriado para o defeito foi avançado e implantado, seguindo as técnicas clássicas já consagradas (Figura 2).

O posicionamento final do dispositivo e a presença de fluxos residuais foram avaliados principalmente pela ecocardiografia e também pela angiografia nos casos realizados no laboratório de cateterismo. Confir-

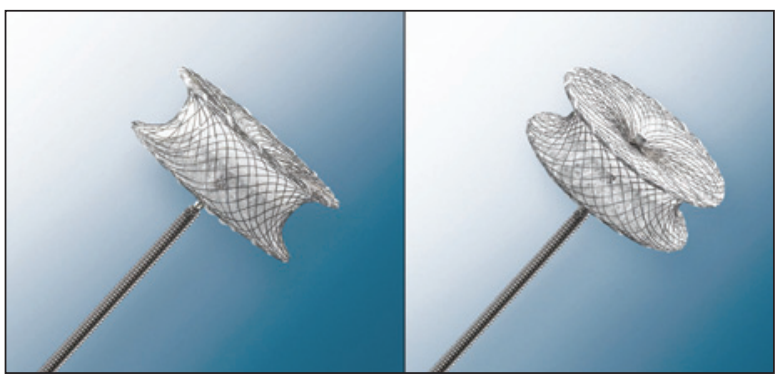

Figura 1 - Prótese Amplatzer de comunicação interventricular muscular. Prótese feita de malha fina de nitinol com dois discos conectados por uma cintura central de $7 \mathrm{~mm}$ de extensão. Os discos têm diâmetro $8 \mathrm{~mm}$ maior que a cintura, que dá o tamanho do dispositivo $(4-16 \mathrm{~mm})$. Internamente, notam-se os retalhos de poliéster. Um cabo de aço de liberação conecta-se ao disco direito por um mecanismo de rosca.

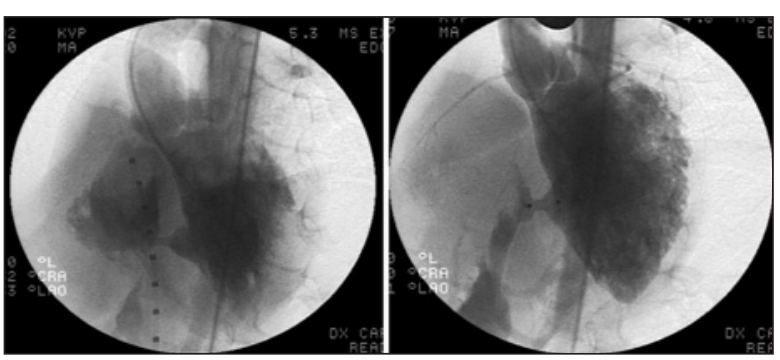

Figura 2 - Implante percutâneo. Painel esquerdo: Ventriculografia esquerda em projeção hepatoclavicular demonstrando comunicação muscular única no meio do septo de $4-5 \mathrm{~mm}$ de diâmetro mínimo com fluxo esquerdo-direito significativo. Painel direito: Ventriculografia após o implante demonstrando dispositivo bem posicionado com fluxo residual mínimo pela malha da prótese imediatamente após o implante. mado o posicionamento correto, a prótese foi liberada e novas avaliações ecocardiográficas e angiográficas foram realizadas.

\section{Avaliação pós-procedimento}

Após o procedimento, a hemostasia foi obtida por meio de compressão manual das regiões inguinais e/ou do pescoço. Os pacientes foram acordados e extubados na sala de cateterismo, sendo transferidos para a unidade de terapia semi-intensiva para monitoração rotineira. No dia seguinte ao procedimento, foram submetidos a exame clínico rotineiro, com obtenção de exames laboratoriais, incluindo eletrocardiograma, radiografia de tórax e ecocardiografia transtorácica para avaliação do posicionamento da prótese e presença de possíveis fluxos residuais. Aqueles que estavam bem clinicamente e sem complicações receberam alta hospitalar com orientação para tomar aspirina (dose de 3-5 mg/kg/dia) por 6 meses e para seguir as recomendações clássicas para profilaxia de endocardite quando necessário nesse período.

O seguimento clínico constou de exame físico, realização de radiografia de tórax, eletrocardiograma, e ecocardiografia transtorácica com Doppler em cores 1 , 6 e 12 meses após implante e, a partir daí, anualmente.

\section{Análise estatística}

Os valores são expressos em freqüências e porcentagens, média e desvio padrão ou mediana e variação conforme distribuição da amostra.

\section{RESULTADOS}

\section{População e características dos defeitos}

De setembro de 2002 a dezembro de 2007, foram realizados 9 procedimentos em 8 pacientes (Tabela 1). A mediana de idade foi de 6 anos (3,5 a 25 anos) e de peso, de $26 \mathrm{~kg}(18-90 \mathrm{~kg})$. Os pacientes apresentavam-se bem clinicamente, controlados com pouca ou nenhuma medicação anticongestiva. Em relação às enfermidades associadas, um paciente de 18 anos era portador de coartação da aorta e foi submetido a implante de stent 10 meses antes do fechamento percutâneo. Outro paciente apresentava PCA e foi submetido a oclusão com mola de Gianturco um ano antes do fechamento da CIV.

Todos os pacientes eram portadores de CIV muscular única, sendo 7 localizadas na porção trabecular média e 1, na porção anterior. A média do maior diâmetro das CIVs foi de 6,0 $\pm 2,1 \mathrm{~mm}$, variando de $4,9 \mathrm{~mm}$ a $9,3 \mathrm{~mm}$.

Todos os pacientes possuíam pressões normais ou discretamente elevadas na artéria pulmonar.

\section{Procedimentos}

Dos 9 procedimentos, utilizou-se o dispositivo Amplatzer de CIV muscular em 7. Os dispositivos Amplatzer de CIA e Amplatzer de PCA foram utilizados 
TABELA 1

Características dos pacientes, dos defeitos e dos procedimentos e desfechos ecocardiográficos

\begin{tabular}{|c|c|c|c|c|c|c|c|c|c|c|}
\hline Paciente & $\begin{array}{l}\text { Idade } \\
\text { (anos) }\end{array}$ & $\begin{array}{c}\text { Peso } \\
\text { (kg) }\end{array}$ & $\begin{array}{l}\text { Local da } \\
\text { CIV }\end{array}$ & $\begin{array}{l}\text { Tamanho da } \\
\text { CIV (mm) }\end{array}$ & $\begin{array}{c}\text { Defeitos } \\
\text { associados }\end{array}$ & Via & Prótese & Complicações & $\begin{array}{c}\text { Fluxo } \\
\text { residual } \\
\text { imediato }\end{array}$ & $\begin{array}{c}\text { Fluxo } \\
\text { residual } \\
\text { tardio }\end{array}$ \\
\hline 1 & 6 & 27 & Trabecular média & 5,1 & PCA & Jugular & $\begin{array}{l}\text { PCA 8-6 } \\
\text { CIV } 8\end{array}$ & $\begin{array}{c}\text { Embolização } \\
\text { Primeira } \\
\text { tentativa }\end{array}$ & Trivial & Não \\
\hline 2 & 12 & 53 & Trabecular média & 9,3 & - & Jugular & CIA 12 & - & Trivial & Não \\
\hline 3 & 17 & 59 & Trabecular média & 5,0 & $\mathrm{CoA}$ & Jugular & CIV 6 & - & Não & Não \\
\hline 4 & 25 & 90 & Trabecular média & 4,9 & - & Jugular & CIV 6 & BRE & Não & Não \\
\hline 5 & 3,5 & 18 & Anterior & 5,8 & - & Femoral & CIV 8 & - & Trivial & Não \\
\hline 6 & 7 & 28 & Trabecular média & 6,3 & - & Jugular & CIV 8 & BRD & Não & Não \\
\hline 7 & 6 & 25 & Trabecular média & 6,1 & - & Jugular & CIV 8 & - & Não & Não \\
\hline 8 & 5 & 20 & Trabecular & 5,5 & - & Jugular & CIV 8 & - & Trivial & Não \\
\hline
\end{tabular}

$\mathrm{BRD}=$ bloqueio de ramo direito; $\mathrm{BRE}=$ bloqueio de ramo esquerdo; $\mathrm{CI} \mathrm{A}=$ comunicação interatrial; $\mathrm{CIV}=$ comunicação interventricular; $\mathrm{CoA}=$ coartação da aorta; $\mathrm{PCA}=$ persistência do canal arterial.

em um caso cada. Em um paciente de 6 anos, portador de CIV muscular trabecular (primeiro paciente da série), houve embolização de um dispositivo Amplatzer de PCA para a aorta descendente, que foi resgatado com técnicas percutâneas sem complicações. Esse paciente voltou um ano depois para novo procedimento, que foi completado com sucesso e sem complicações com uma prótese Amplatzer de CIV muscular. $\mathrm{O}$ restante dos procedimentos foi realizado com sucesso. Um paciente apresentou morfologia de bloqueio de ramo direito e outro de bloqueio de ramo esquerdo dentro das primeiras 24 horas após o implante. Não houve indução de arritmias ou de bloqueio atrioventricular total.

Logo após o implante, 4 pacientes apresentavam oclusão do defeito pela ecocardiografia transesofágica e os 4 restantes apresentavam fluxos residuais discretos pela malha da prótese Amplatzer. Todos os pacientes tiveram alta no dia seguinte ao procedimento, 7 dos 8 pacientes já apresentando oclusão total à ecocardiografia.

\section{Seguimento}

O seguimento clínico variou de 9 meses a 4 anos, com mediana de 2 anos. A taxa de oclusão pela ecocardiografia foi de $100 \%$ (8/8). No seguimento, todos os pacientes encontravam-se em ritmo sinusal e apresentavam ventrículo esquerdo com volume diastólico final de dimensões normais à ecocardiografia. Não houve episódios tardios de embolização, endocardite, arritmias, perfurações ou disfunção das valvas atrioventriculares e semilunares. No paciente com coartação da aorta, não houve sinais clínicos nem ecocardiográficos de recoartação, e no paciente com PCA não houve recanalização do mesmo.

\section{DISCUSSÃO}

Apesar de não serem tão comuns, as CIVs musculares congênitas constituem-se em desafio técnico ao cirurgião, que tem dificuldade para localizá-las no meio das trabeculações grosseiras do ventrículo direito. Conseqüentemente, é freqüente a ocorrência de fluxos residuais, muitas vezes requerendo reoperações ${ }^{2-6}$. As CIVs musculares apicais também são de difícil abordagem cirúrgica pelo ventrículo direito, muitas vezes necessitando de ventriculotomia esquerda, que resulta em disfunção ventricular e complicações no pós-operatório ${ }^{2-6}$. Tais problemas são exacerbados em pequenos lactentes, o que pode levar a índices de mortalidade não desprezíveis ${ }^{2-6}$. Nesses casos, a bandagem da artéria pulmonar pode ser uma opção técnica inicial mais segura, porém tem caráter paliativo, já que compromete o paciente a procedimentos repetidos no futuro. Além disso, todas as técnicas cirúrgicas, à exceção da bandagem da artéria pulmonar, requerem uso de circulação extracorpórea, com suas conhecidas complicações sobre os diversos sistemas do organismo. Entre as mais temidas encontram-se as crises de hipertensão pulmonar no pós-operatório, principalmente em pequenos lactentes com grandes CIVs, e, possivelmente, a redução do coeficiente de inteligência e comprometimento neurológico a longo prazo ${ }^{26-28}$. Adicionalmente, a abordagem cirúrgica necessita de toracotomia, resultando em cicatriz indelével para o resto da vida.

Pelos motivos expostos, vários investigadores procuraram alternativas menos invasivas para oclusão desses defeitos. Lock et al. ${ }^{7}$ foram os primeiros a comprovar a exeqüibilidade do método percutâneo em uma pequena série de pacientes com CIVs musculares pósinfarto, publicada no final da década de 80. O método foi posteriormente estendido para pacientes portado- 
res de CIVs musculares congênitas, com uso de dispositivos tipo duplo disco, resultando em índices de oclusão subótimos ${ }^{9,10}$. Ficava clara a necessidade de um dispositivo especialmente desenhado para oclusão desse tipo de defeito. Amin et al. ${ }^{29}$ foram os primeiros a relatar o uso de um dispositivo de nitinol da família Amplatzer com características específicas para oclusão da CIV muscular em um modelo experimental, em 1998. Tal relato foi seguido pela primeira experiência bem-sucedida realizada em humanos e publicada por Thanopoulos et al. ${ }^{11}$, em 1999. Outros autores subseqüentemente publicaram suas experiências, demonstrando resultados consistentes e reprodutíveis em diversos centros no mundo ${ }^{12-19}$, inclusive em nosso meio $^{23}$.

São várias as vantagens das próteses da família Amplatzer, a saber: mecanismo de implante simples e de fácil familiarização pelo intervencionista; necessidade de sistemas de baixo perfil; autocentralização dada pela cintura central, que preenche o defeito e exerce efeito de stent em suas bordas; e, finalmente, ser totalmente recapturável e reposicionável, com alto poder oclusor. Tais propriedades são, em grande parte, responsáveis pelos altos índices de sucesso técnico e de oclusão e pelos baixos índices de complicação encontrados neste e em outros estudos.

Apesar de ser relativamente simples, a técnica de implante percutâneo é algo trabalhosa e, a nosso ver, é recomendável que o procedimento seja realizado ou supervisionado por intervencionistas pediátricos com experiência em oclusão de outros defeitos cardíacos e que estejam familiarizados com dispositivos intracardíacos e com imagens ecocardiográficas. Mais especificamente, durante a formação da alça arteriovenosa, é necessário estar seguro sobre o funcionamento adequado da valva tricúspide ${ }^{30}$. No caso de mau funcionamento dessa valva, em alguns casos pode ser necessário cruzar novamente o defeito para se estabelecer outra vez a alça arteriovenosa com o fio-guia. Além disso, a tensão nos guias e cateteres pode resultar em instabilidade hemodinâmica, principalmente em lactentes jovens com menos de $8 \mathrm{~kg}$, durante o implante percutâneo no laboratório de cateterismo ${ }^{14,22}$. Por esse motivo, a técnica perventricular, não estudada nesta série de pacientes, é de extrema utilidade para o manejo desses defeitos nesse subgrupo de doentes. Inicialmente relatada por Amin et al. ${ }^{31}$, em 1998, outros autores popularizaram essa forma de abordagem híbrida, que viabiliza a oclusão de grandes defeitos em pequenos pacientes ${ }^{22,31-34}$. Nessa pequena série de pacientes com mais de 5 anos com CIVs restritivas, o procedimento se mostrou muito seguro, com baixos índices de complicações. O episódio de embolização ocorreu em nosso primeiro caso e, provavelmente, também esteve associado à escolha inadequada do dispositivo de PCA. Naquela ocasião, ainda não estavam disponíveis os dispositivos de duplo disco de CIV muscular. Mesmo assim, a prótese embolizada para a aorta foi resgatada por técnicas percutâneas sem complicações, tendo sido realizado novo procedimento, com sucesso, um ano após. O encontro de morfologias de bloqueio de ramos direito e esquerdo neste estudo também foi relatada em outras experiên$\operatorname{cias}^{35}$. Tais alterações são freqüentemente observadas no pós-operatório da correção cirúrgica desses defeitos e não trazem maiores conseqüências. A ocorrência de bloqueio atrioventricular total após o fechamento percutâneo da CIV muscular é excepcional ${ }^{17}$, já que os defeitos musculares se encontram distantes do nó atrioventricular. Os estudos experimentais demonstram que esses dispositivos, mesmo no septo interventricular, levam cerca de 3 a 6 meses para ser endotelizados $^{29}$. Por isso, recomenda-se o uso de antiplaquetários durante esse período.

Neste estudo com número limitado de pacientes, observamos eficácia de $100 \%$ para oclusão dos defeitos. Em outros estudos com maior número de pacientes, altos índices de oclusão (> 90\%) também foram encontrados ${ }^{11-19,22}$. Tais taxas são, no mínimo, comparáveis às taxas cirúrgicas, sendo, provavelmente, meIhores. Devido à segurança e à eficácia do uso da prótese Amplatzer para oclusão da CIV muscular congênita, o Food and Drug Administration (FDA), recentemente, aprovou a utilização desse dispositivo para uso clínico (agosto de 2007).

Apesar de não ter sido o foco deste estudo, esse tipo de dispositivo também pode ser aplicado para oclusão de CIVs musculares múltiplas ${ }^{14,17}$. Entretanto, o custo associado à utilização de múltiplas próteses é um fator limitante, principalmente em nosso meio. Outras aplicações clínicas incluem ainda a oclusão de CIVs residuais pós-cirúrgicas ${ }^{36,37}$, de CIVs traumáticas ${ }^{38,39} \mathrm{e}$ de CIVs pós-infarto ${ }^{40}$.

Em conclusão, este estudo demonstrou a exeqüibilidade, a segurança e a eficácia da oclusão percutânea da CIV muscular única restritiva de etiologia congênita em uma série pequena de pacientes não-consecutivos com mais de 5 anos de idade. Mesmo reconhecendo que seria necessário maior número de pacientes para uma análise mais precisa da segurança do método, é provável que, com o acúmulo de experiência, a incidência de complicações, que já é baixa, tenda a diminuir ainda mais. Além disso, o tempo de seguimento dos pacientes é ainda limitado (mediana de 2 anos). Entretanto, baseado nos resultados a longo prazo com próteses tipo Amplatzer para oclusão de $\mathrm{ClA}$ e de outras próteses para oclusão da CIV muscular, é improvável que complicações tardias ocorram. Estudos prospectivos randomizados comparando as técnicas cirúrgicas e percutâneas estão ausentes na literatura e dificilmente serão realizados. Mesmo com essas limitações, acreditamos que os resultados observados neste estudo e em outros disponíveis na literatura nos permitam afirmar que os métodos aqui descritos devam ser escoIhidos como os de eleição para o manejo de pacientes com CIV muscular única de etiologia congênita. 


\section{REFERÊNCIAS BIBLIOGRÁFICAS}

1. Tynan M, Anderson RH. Ventricular septal defect. In: Anderson $\mathrm{RH}$, Baker EJ, Macartney FJ, Rigby ML, Shinebourne EA, Tynan M, editors. Paediatric cardiology. London: Churchill Livingstone; 2002. p.983-1014.

2. Kouchoukos NT, Blackstone EH, Doty DB, Hanley FL, Karp RB. Ventricular septal defect. In: Kouchoukos NT, Blackstone EH, Doty DB, Hanley FL, Karp RB, editors. Kirklin/BarratBoyes: Cardiac surgery. Philadelphia: Churchill Livingstone; 2003. p.850-910.

3. Serraf A, Lacour-Gayet F, Bruniaux J, Quaknine R, Losay J, Petit J, et al. Surgical management of isolated multiple ventricular septal defects. Logical approach in 130 cases. J Thorac Cardiovasc Surg. 1992;103(3):437-42.

4. Kitagawa T, Durham LA $3^{\text {rd }}$, Mosca RS, Bove EL. Techniques and results in the management of multiple ventricular septal defects. J Thorac Cardiovasc Surg. 1998;115(4):848-56.

5. Hanna B, Colan SD, Bridges ND, Mayer JE, Castaneda AR. Clinical and myocardial status after left ventriculotomy for ventricular septal defect closure. J Am Coll Cardiol. 1991;17 (Suppl):110A.

6. Wollenek G, Wyse R, Sullivan I, Elliott M, de Leval M, Stark J. Closure of muscular ventricular septal defects through a left ventriculotomy. Eur J Cardiothorac Surg. 1996;10(8):595-8.

7. Lock JE, Block PC, McKay RG, Baim DS, Keane JF. Transcatheter closure of ventricular septal defects. Circulation. 1988;78(2):361-8.

8. Bridges ND, Perry SB, Keane JF, Goldstein SA, Mandell V, Mayer JE Jr, et al. Preoperative transcatheter closure of congenital muscular ventricular septal defects. N Engl J Med. 1991;324(19):1312-7.

9. Sideris EB, Walsh KP, Haddad JL, Chen CR, Ren SG, Kulkarni $\mathrm{H}$. Occlusion of congenital ventricular septal defects by the buttoned device. "Buttoned device" Clinical Trials International Register. Heart. 1997;77(3):276-9.

10. Janorkar S, Goh T, Wilkinson J. Transcatheter closure of ventricular septal defects using the Rashkind device: initial experience. Catheter Cardiovasc Interv. 1999;46(1):43-8.

11. Thanopoulos BD, Tsaousis GS, Konstadopoulou GN, Zarayelyan AG. Transcatheter closure of muscular ventricular septal defects with the Amplatzer ventricular septal defect occluder: initial clinical applications in children. J Am Coll Cardiol. 1999;33(5):1395-9.

12. Hijazi ZM, Hakim F, Al-Fadley F, Abdelhamid J, Cao QL. Transcatheter closure of single muscular ventricular septal defects using the Amplatzer muscular VSD occluder: initial results and technical considerations. Catheter Cardiovasc Interv. 2000;49(2):167-72.

13. Hijazi ZM. Device closure of ventricular septal defects. Catheter Cardiovasc Interv. 2003;60(1):107-14.

14. Holzer R, Balzer D, Cao QL, Lock K, Hijazi ZM. Device closure of muscular ventricular septal defects using the Amplatzer muscular ventricular septal defect occluder: immediate and mid-term results of a U.S. registry. J Am Coll Cardiol. 2004;43(7):1257-63.

15. Arora R, Trehan V, Thakur AK, Mehta V, Sengupta PP, Nigam M. Transcatheter closure of congenital muscular ventricular septal defect. J Interv Cardiol. 2004;17(2):109-15.

16. Thanopoulos BD, Rigby ML. Outcome of transcatheter closure of muscular ventricular septal defects with the Amplatzer ventricular septal defect occluder. Heart. 2005;91(4):513-6.

17. Carminati M, Butera G, Chessa M, Drago M, Negura D, Piazza L. Transcatheter closure of congenital ventricular septal defect with Amplatzer septal occluders. Am J Cardiol. 2005;96(12A):52L-58L.
18. Djer MM, Latiff HA, Alwi M, Samion H, Kandavello G. Transcatheter closure of muscular ventricular septal defect using the Amplatzer devices. Heart Lung Circ. 2006;15(1):12-7.

19. Butera G, Chessa M, Carminati M. Percutaneous closure of ventricular septal defects. Cardiol Young. 2007;17(3):243-53.

20. Knauth AL, Lock JE, Perry SB, McElhinney DB, Gauvreau K, Landzberg MJ, et al. Transcatheter device closure of congenital and postoperative residual ventricular septal defects. Circulation. 2004;110(5):501-7.

21. Lim DS, Forbes TJ, Rothman A, Lock JE, Landzberg MJ. Transcatheter closure of high-risk muscular ventricular septal defects with the CardioSEAL occluder: initial report from the CardioSEAL VSD registry. Catheter Cardiovasc Interv. 2007;70(5):740-4.

22. Diab KA, Cao QL, Mora BN, Hijazi ZM. Device closure of muscular ventricular septal defects in infants less than one year of age using the Amplatzer devices: feasibility and outcome. Catheter Cardiovasc Interv. 2007;70(1):90-7.

23. Queiroz FJ, Rossi Filho RI, Ramos S, Esteves C, Queiroz DS, Machado PR, et al. Percutaneous occlusion of interventricular septal defects. Initial experiment. Arq Bras Cardiol. 2005;85(3):174-9.

24. Pedra CA, Pedra SR, Esteves CA, Pontes SC Jr, Braga SL, Arrieta SR, et al. Percutaneous closure of perimembranous ventricular septal defects with the Amplatzer device: technical and morphological considerations. Catheter Cardiovasc Interv. 2004;61(3):403-10.

25. Pedra CA, Pedra SR, Esteves CA, Chamie F, Christiani LA, Fontes VF. Transcatheter closure of perimembranous ventricular septal defects. Expert Rev Cardiovasc Ther. 2004;2(2):253-64.

26. Kaltman JR, Jarvik GP, Bernbaum J, Wernovsky G, Gerdes M, Zackai $\mathrm{E}$, et al. Neurodevelopmental outcome after early repair of a ventricular septal defect with or without aortic arch obstruction. J Thorac Cardiovasc Surg. 2006;131(4):792-8.

27. Hövels-Gürish HH, Konrad K, Skorzenski D, HerpertzDahlmann B, Messmer BJ, Seghaye MC. Attentional dysfunction in children after corrective cardiac surgery in infancy. Ann Thorac Surg. 2007;83(4):1425-30.

28. Hövels-Gürish HH, Konrad K, Skorzenski D, Minkenberg R, Herpertz-Dahlmann B, Messmer BJ, et al. Long-term behavior and quality of life after corrective cardiac surgery in infancy for tetralogy of Fallot or ventricular septal defect. Pediatr Cardiol. 2007;28(5):346-54

29. Amin Z, Gu X, Berry JM, Bass JL, Titus JL, Urness M, et al. New device for closure of muscular ventricular septal defects in a canine model. Circulation. 1999;100(3):320-8.

30. Christiani LA, Bergman F, Tress JC, Vanzillotta PP, Pedra CA. Severe tricuspid stenosis during percutaneous occlusion of perimembranous ventricular septal defect with the new Amplatzer device. Congenit Heart Dis. 2006;1(5):239-43.

31. Amin Z, Berry JM, Foker JE, Rocchini AP, Bass JL. Intraoperative closure of muscular ventricular septal defect in a canine model and application of the technique in a baby. J Thorac Cardiovasc Surg. 1998;115(6):1374-6.

32. Bacha EA, Cao QL, Starr JP, Waight D, Ebeid MR, Hijazi ZM. Perventricular device closure of muscular ventricular septal defects on the beating heart: technique and results. J Thorac Cardiovasc Surg. 2003;126(6):1718-23.

33. Bacha EA, Cao QL, Galantowicz ME, Cheatham JP, Fleishman CE, Weinstein SW, et al. Multicenter experience with perventricular device closure of muscular ventricular septal defects. Pediatr Cardiol. 2005;26(2):169-75.

34. Diab KA, Hijazi ZM, Cao QL, Bacha EA. A truly hybrid approach to perventricular closure of multiple muscular ventricular septal defects. J Thorac Cardiovasc Surg. 2005. 130(3):892-3. 
35. Robinson JD, Zimmerman FJ, De Loera O, Heitschmidt $M$, Hijazi ZM. Cardiac conduction disturbances seen after transcatheter device closure of muscular ventricular septal defects with the Amplatzer occluder. Am J Cardiol. 2006;97(4):558-60.

36. Walsh MA, Coleman DM, Oslizlok P, Walsh KP. Percutaneous closure of postoperative ventricular septal defects with the Amplatzer device. Catheter Cardiovasc Interv. 2006;67(3):445-51

37. Pedra CA, Pontes SC Jr, Pedra SR, Salerno L, Sousa JB, Miaira $M A$, et al. Percutaneous closure of postoperative and posttraumatic ventricular septal defects. J Invasive Cardiol. $2007 ; 19(11): 491-5$.
38. Bauriedel G, Redel DA, Schmitz C, Welz A, Schild HH, Lüderitz B. Transcatheter closure of a posttraumatic ventricular septal defect with an Amplatzer occluder device. Catheter Cardiovasc Interv. 2001;53(4):508-12.

39. Cowley CG, Shaddy RE. Transcatheter treatment of a large traumatic ventricular septal defect. Catheter Cardiovasc Interv. 2004;61(1):144-6.

40. Holzer R, Balzer D, Amin Z, Ruiz CE, Feinstein J, Bass J, et al. Transcatheter closure of postinfarction ventricular septal defects using the new Amplatzer muscular VSD occluder: Results of a U.S. Registry. Catheter Cardiovasc Interv. 2004;61(2):196-201. 